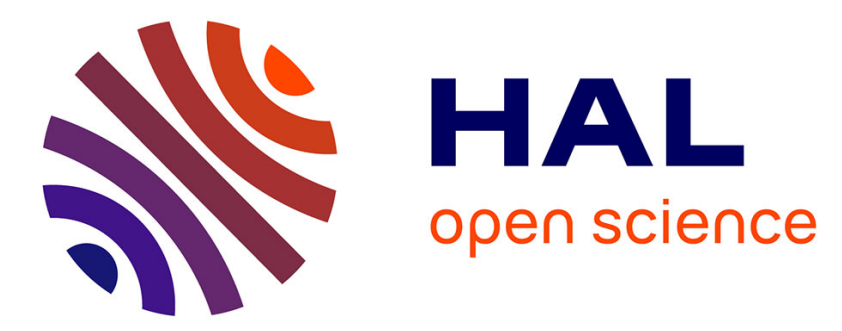

\title{
Structure des multicouches métalliques et de leurs interfaces vue par RMN
}

P. Panissod, Jean-Philippe Jay, C. Meny, M. Wojcik, E. Jedryka

\section{To cite this version:}

P. Panissod, Jean-Philippe Jay, C. Meny, M. Wojcik, E. Jedryka. Structure des multicouches métalliques et de leurs interfaces vue par RMN. Journal de Physique IV Proceedings, 1996, 06 (C7), pp.C7-89-C7-106. 10.1051/jp4:1996711 . jpa-00254499

\section{HAL Id: jpa-00254499 https://hal.science/jpa-00254499}

Submitted on 1 Jan 1996

HAL is a multi-disciplinary open access archive for the deposit and dissemination of scientific research documents, whether they are published or not. The documents may come from teaching and research institutions in France or abroad, or from public or private research centers.
L'archive ouverte pluridisciplinaire HAL, est destinée au dépôt et à la diffusion de documents scientifiques de niveau recherche, publiés ou non, émanant des établissements d'enseignement et de recherche français ou étrangers, des laboratoires publics ou privés. 


\title{
Structure des multicouches métalliques et de leurs interfaces vue par RMN
}

\author{
P. Panissod, C. Meny, J.Ph. Jay, M. Wojcik* et E. Jedryka* \\ Institut de Physique et Chimie des Matériaux de Strasbourg, CNRS-ULP, 23 rue du Loess, \\ 67037 Strasbourg, France \\ * Institute of Physics, Polish Academy of Sciences, Al. Lotnikow 32-46, 02668 Warsaw, Poland
}

\begin{abstract}
Résumé : En raison de la sensibilité du champ hyperfin à l'environnement des noyaux sondés, les spectres RMN peuvent être considérés comme des histogrammes détaillés de l'ordre chimique à courte distance présent dans les matériaux étudiés. En complément des techniques standards de diffraction, cette technique fournit ainsi une vue locale de la structure dans l'espace direct. Nous en présentons ici les développements récents appliqués à l'étude de la structure de multicouches $\mathrm{Co} / \mathrm{X}$ et nous montrons comment la modélisation des spectres permet une description détaillée et quantitative de la façon selon laquelle les deux éléments se mélangent, éventuellement, aux interfaces. Les multicouches. $\mathrm{Co} / \mathrm{Cu}$, deux eléments peu miscibles, ont été spécialement étudiées. Ce cas est le plus favorable pour l'obtention d'interfaces abruptes mais, en fait, selon les conditions et des techniques de préparation, les interfaces peuvent être très différentes, abruptes, rugueuses ou même très mélangées. Avec des eléments formant des solutions solides $(\mathrm{Co} / \mathrm{Ru}$ ou $\mathrm{Co} / \mathrm{Cr}$ ), pour lesquels une interdiffusion est plus probable, on observe aux interfaces un mélange sur plusieurs distances atomiques mais les profils de diffusion s'avèrent beaucoup moins dépendants des conditions de préparation que dans le cas précédent. Enfin les éléments formant des composés intermétalliques $(\mathrm{Co} / \mathrm{Fe})$ présentent le plus fort mélange aux interfaces avec une structure qui semble réminiscente du diagramme de phase massif.
\end{abstract}

\section{INTRODUCTION}

L'influence de la structure détaillée des multicouches et sandwiches métalliques sur leurs propriétés magnétiques et de transport est bien établie. Plus critique encore est la structure des interfaces compte tenu du fait que les nouvelles propriétés observées dans ces systèmes résultent, précisément, de leur forte densité d'interfaces. Il est donc très important que soit connue de manière détaillée la morphologie des interfaces et, en particulier, de pouvoir distinguer entre un mélange interfacial résultant d'une rugosité à longue distance de celui résultant d'une interdiffusion à courte distance ou même de la formation d'un composé intermétallique. La sensibilité du champ hyperfin à l'environnement chimique (nombre et nature des voisins) ainsi qu'à la topologie (distances et symétrie ponctuelle) peut être employée pour l'étude de la structure atomique locale des couches et de leur interfaces. Dans cet article nous mettons l'accent seulement sur l'interaction hyperfine magnétique. L'interaction quadripolaire électrique s'avère très utile également pour les études structurales en tant que signature de configurations atomiques et pour la détermination de la symétrie ponctuelle. Cependant, dans les multicouches magnétiques, elle est apparemment trop faible en regard de l'interaction magnétique pour produire des effets considérables.

L'information structurale à l'échelle atomique que l'on obtient par RMN est tout à fait complémentaire de celle fournie par les diffraction standards, rayons X, neutrons ou électrons. En terme de distance sondée et de sélectivité élémentaire la RMN peut être comparée à la technique EXAFS: moins quantitative que l'EXAFS en ce qui concerne les distances, elle fournit, en revanche, des informations beaucoup plus détaillées sur les distributions de configurations chimiques locales. En effet la distribution du champ hyperfin sur un noyau -le spectre RMN- reflète directement la distribution de probabilité d'oc- 
currence des configurations de premiers voisins de ce noyau: chaque configuration donne lieu, dans le spectre, à une raie caractéristique dont l'intensité est proportionnelle à l'abondance de cette configuration. Cette distribution peut ensuite être comparée à la distribution théorique résultant d'une structure particulière d'interface telles une interface abrupte parfaite, une interface abrupte contenant des marches monoatomiques, une interface fortement interdiffusée ou encore une interface discontinue ou couche granulaire. De cette comparaison, la nature du mélange aux interfaces (solution solide, ségrégation ou composé intermétallique) peut être également déduite de l'observation et de la simulation. Quantitativement on peut ainsi évaluer les profils de concentration avec une résolution atomique, la densité de marches, la taille moyenne de grains ou d'îlots, ce qui permet de caractériser la rugosité d'interface à l'échelle des distances interatomiques. En revanche une rugosité à longue distance ( $>10$ distances atomiques), telle qu'en présente une interface abrupte mais ondulée, sera perçue comme parfaite par RMN quand les diffractions standards verront encore cette rugosité comme un mélange. Outre les interfaces, d'autres surfaces internes telles que les joints de grains peuvent être observées par RMN et, bien sûr, la technique permet également de caractériser la structure cristallographique de la partie massive des couches (par exemple les phases cfc ou hc ou même la phase métastable cc du cobalt) et ses défauts.

Si l'on considère, de plus, que les données primaires d'une observation RMN sont de nature magnétique, la technique est particulièrement adaptée à l'étude des corrélations entre la microstructure des multicouches et leur propriétés magnétiques.

La méthode a été développée et appliquée principalement à des multicouches à base de cobalt. Dans la première partie de cet article nous présentons une introduction à l'analyse des spectres et aux divers modèles de structure d'interfaces qui ont été confrontés aux résultats expérimentaux. La seconde partie est consacrée à l'illustration sur des exemples couvrant les cas de métaux qui, dans l'état massif, sont non miscibles, ou forment des solutions solides, ou enfin forment des composés intermétalliques.

\section{METHODES: ANALYSE DES SPECTRES ET MODELES D'INTERFACE}

La difficulté des investigations structurales par RMN provient du caractère indirect de l'information contenue dans le spectre: la RMN sonde le magnétisme local (champ hyperfin - moment magnétique) autour des noyaux observés lequel détermine la position de la raie de résonance. Ce magnétisme local est, quant à lui, dépendant de la structure et de l'environnement local. Dans les systèmes dont on connaît la structure, les mesures RMN renseignent sur la relation entre champ hyperfin et coordination atomique locale. Réciproquement, les études structurales par RMN nécessitent de connaître a priori cette relation avant de pouvoir tirer, de l'analyse des spectres, toute information sur les configurations atomiques présentes dans un système inconnu. Dans les composés organiques, objets de choix pour la RMN analytique, les spectres de référence sont connus expérimentalement et théoriquement pour la plupart des radicaux en raison du faible nombre d'éléments et de configurations moléculaires impliqués. Malheureusement, dans les alliages et composés métalliques ferromagnétiques, les calculs ab initio du champ hyperfin, en dépit de progrès significatifs récents [1], ne peuvent encore fournir la relation détaillée entre le champ hyperfin et les multiples configurations atomiques locales intervenant dans des systèmes pouvant être localement inhomogènes. C'est pourquoi un protocole obligé consiste, dans une première étape, en l'étude d'échantillons de référence -alliages ou composés ordonnés- de structure bien connue, de manière à obtenir expérimentalement l'information nécessaire sur les propriétés hyperfines locales (c.a.d. établir la relation, biunivoque autant que possible, entre raie spectrale -fréquenceet configuration atomique locale). Dans une seconde étape cette information permet de déterminer, dans les systèmes de structure inconnue, les probabilités de présence des différentes phases, ou configurations atomiques, ou environnements locaux. L'étape finale, et le but ultime de toute étude structurale, est de proposer un arrangement tridimensionnel des atomes dont l'ordre chimique et topologique à courte distance soit compatible avec l'observation expérimentale, sachant toutefois que la remontée de l'échelle locale -ponctuelle- vers le tridimensionnel n'est pas univoque. 


\subsection{Etudes des références massives}

Dans les systèmes métalliques magnétiques le champ hyperfin (HF) est déterminé par la structure électronique (moment magnétique local et polarisation des électrons de conduction). C'est pourquoi, autant que faire se peut, les références doivent avoir une structure électronique proche de ces systèmes bimétalliques que sont les multicouches magnétiques. Pratiquement le choix est limité aux alliages et composés massifs et il n'est pas évident, a priori, que la condition de similarité de structure électronique soit satisfaite entre alliages et interfaces bimétalliques. La validité de telles références sera discutée à la fin de cette section.

Dans les alliages, la distribution de champ hyperfin due au désordre chimique résulte en des spectres formés de plusieurs raies (satellites): chaque atome étranger dans la couche de premiers voisins (PV) du noyau sondé modifie le HF de sorte que chaque configuration de PV donne lieu à une raie différente dans le spectre. Dans la plupart des cas l'effet des atomes étrangers PV est pratiquement additif et les raies satellites sont presque également espacées. L'influence des couches de voisins plus éloignés diminue rapidement avec la distance et ils ne contribuent qu'à un élargissement des satellites principaux. Des satellites résolus dus à des impuretés sur les couches éloignées ne peuvent être observés que pour de très faibles concentrations. Ainsi un spectre d'alliage consiste généralement en une succession de raies régulièrement espacées correspondant aux noyaux ayant respectivement $0,1,2, \ldots$ atomes étrangers parmi leur $\mathrm{N}$ premiers voisins. L'intensité relative de chacun de ces satellites est proportionnelle à la probabilité de présence de chacune de ces configurations de PV [2]. Par exemple, pour les bonnes solutions solides, ces probabilités obéissent à une loi binomiale. Sur la figure 1 on peut voir le cas d'un spectre de ${ }^{59} \mathrm{Co}$ dans un alliage $\mathrm{CoCr}$ hexagonal compact. Pour la concentration de $14 \%$ en Cr présentée, la raie la plus intense est celle qui correspond aux noyaux de Co ayant $1 \mathrm{Cr}$ parmi leur $12 \mathrm{PV}$; à plus haute fréquence se situe la raie du cobalt entouré de 12 Co (Co hc pur) et à plus basse fréquence deux autres raies correspondant au Co ayant 2 et $3 \mathrm{Cr} \mathrm{PV}$ puis une queue peu structurée pour les noyaux de Co ayant plus de $3 \mathrm{Cr}$ PV. L'identification de ces raies se fait sans ambiguilté par comparaison, en fonction de la concentration, des intensités expé-

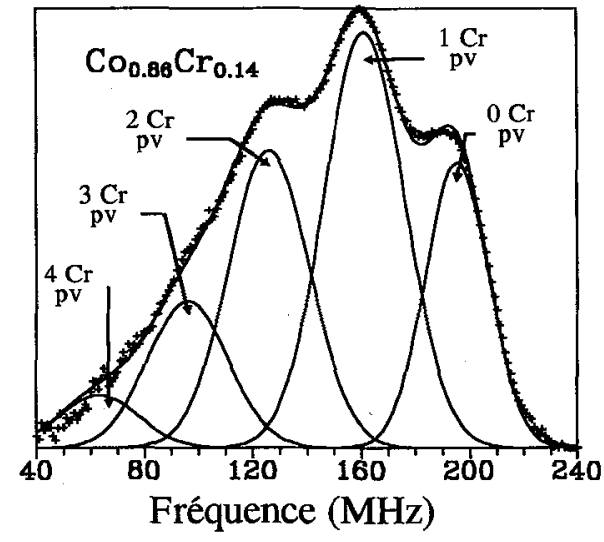

Fig.1. Exemple de spectre du ${ }^{59} \mathrm{Co}$ observé dans un alliage de référence $\mathrm{Co}_{86} \mathrm{Cr}_{14}$ (solution solide) et sa reconstruction avec 5 raies gaussiennes correspondant aux configurations avec $0 \mathrm{Cr}$ à $4 \mathrm{Cr}$ parmi les 12 proches voisins du Co dans la structure hc. rimentales avec les probabilités prédites par la loi binomiale. Sur la même figure on peut voir la reconstruction de ce spectre avec un jeu de 5 raies gaussiennes séparées de $32 \mathrm{MHz}$ correspondant aux configurations de 0 à $4 \mathrm{Cr} \mathrm{PV}$ autour du Co.

L'utilité, en tant que référence, des alliages désordonnés est limitée aux basses concentrations $(<25 \%)$ car la résolution des satellites diminue rapidement lorsque croît le désordre chimique. La positions des raies correspondant à un grand nombre d'atomes étrangers PV est donc mal connue. Les spectres larges et mal résolus observés à plus forte concentration peuvent toujours être analysés en termes de distribution binomiale de raies régulièrement espacées mais, bien sur, avec plus d'incertitude sur la position exacte des raies. La variation du HF moyen avec la composition de l'alliage (c.a.d. le nombre moyen de PV) peut également être utilisée avec les mêmes restrictions. L'espacement quasi linéaire des satellites de bas indice observés pour les alliages peu concentrés peut enfin être extrapolé mais il faut garder en tête que la validité de fortes extrapolations reste douteuse [2]. Par chance un spectre d'interface est, par construction, dominé par les raies liées aux configurations à faible nombre de PV de l'autre élément et toute queue étendue du spectre d'interface contient peu d'information en dehors de son intensité intégrale (la quantité du métal sondé perdue dans l'autre métal). Donc l'analyse des spectres 
relève principalement de l'étude de la position et de l'intensité de raies de bas indices qui sont clairement observées dans les alliages de référence.

Il peut arriver qu'il n'existe pas d'alliage de basse concentration avec la structure cristallographique désirée (c'est le cas, par exemple pour la phase cubique centrée du système $\mathrm{CoFe}$ ). Dans ce cas il est possible de se servir de composés ordonnés comme référence, s'ils existent. En effet des composés ordonnés, préparés autour de la composition stoechiométrique, présenterons des spectres structurés en satellites comme les alliages dilués: dans ce cas ce sont les atomes en excès dans la structure (antisites) qui jouent le rôle des impuretés. La figure 2 présente le spectre d'un composé ordonné $\mathrm{Co}_{1+\mathrm{x}} \mathrm{Fe}_{1-\mathrm{x}}$ hors stoechiométrie. On peut y voir plusieurs satellites résolus à haute fréquence correspondant aux atomes réguliers de Co ayant 0 à 4 atomes de Co en antisite parmi leur $8 \mathrm{PV}$. La raie la plus haute en fréquence $(290 \mathrm{MHz})$ est celle de la structure stoechiométrique B2 parfaite (pas d'antisite Co voisin et $8 \mathrm{Fe}$ voisins). Les satellites sont régulièrement espacés de $10-11 \mathrm{MHz}$, valeur également donnée par l'analyse de la variation du champ hyperfin moyen en fonction de la composition dans les alliages désordonnés. La raie large à basse fréquence $(190 \mathrm{MHz})$ est celle qui correspond aux atomes de Co en excès situés sur le sous-réseau du fer et donc entourés uniquement de Co. La position de cette raie est légè-

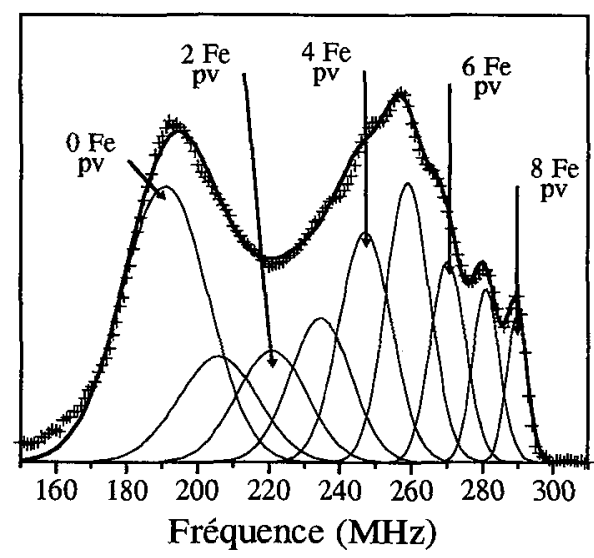

Fig.2. Exemple de spectre ${ }^{59} \mathrm{Co}$ observé dans un échantillon de référence $\mathrm{Co}_{70} \mathrm{Fe}_{30}$ sous stoechiométrique ordonné et sa reconstruction avec 8 gaussiennes correspondant aux coordinations de $0 \mathrm{Fe}$ à $8 \mathrm{Fe}$ parmi les 8 proches voisins dans la phase $\mathrm{B} 2$ cubique centrée. rement plus basse que celle attendue d'une extrapolation d'après la position des raies haute fréquences. Ceci montre les limites de validité d'une extrapolation strictement linéaire. En fait l'analyse détaillée [3] de cette déviation montre qu'elle est due à la différence de configuration sur la deuxième couche de voisins. En effet les atomes de Co sur leur sousréseau n'ont pas ou très peu de Fe en seconds voisins, au contraire, les Co en antisite en ont beaucoup. Il n'est d'ailleurs pas surprenant, dans la structure cc, que les 6 seconds voisins aient une influence non négligeable car ils sont à peine plus éloignés que les $8 \mathrm{PV}$.

Ces deux exemples de références massives montrent clairement que chaque système bimétallique doit être étudié au cas par cas. Une fois établie, par l'étude des références, la relation entre l'évolution du champ hyperfin (de la fréquence de résonance) avec la configuration chimique locale, on peut ensuite utiliser l'information obtenue (positions des satellites ou leur espacement) pour l'analyse de la structure des interfaces. Toutefois cette analyse doit être faite avec précaution: bien que la modification du champ hyperfin soit liée principalement au nombre d'atomes différents dans l'environnement du noyau sondé, le champ hyperfin sur une interface bimétallique parfaite peut être différent de ce qu'il est dans un alliage bimétallique pour la même coordination chimique. Les principales raisons pour lesquelles on peut s'attendre à une différence sont les suivantes. (I) Même s'il y a un fort désordre chimique à l'interface, ce désordre n'est pas isotrope en raison du gradient de concentration [4]. Cette différence peut être importante dans des structures cristallographiques anisotropes (hexagonale ou tétragonale). (II) $S$ 'il y a une désadaptation du paramètre de réseau entre les deux métaux -ce qui est fréquemment le cas- les couches peuvent être contraintes et les distances atomiques différentes de ce qu'elles sont dans les alliages [5]. De plus les contraintes peuvent aussi introduire une anisotropie, par exemple une distorsion tétragonale dans un cristal initialement cubique. (III) Une dernière mais moindre différence peut être un petit déplacement de tout le spectre du système inconnu par rapport à celui observé dans un alliage massif, déplacement dû aux différences d'aimantation moyenne et du champ dipolaire. Ces points seront illustrés dans la section 3. Heureusement toutes ces différences ont peu de chances d'introduire des erreurs d'interprétation dans une analyse soignée des interfaces "réelles". En effet les références ne 
sont strictement nécessaires que dans les cas d'interfaces très mélangées pour lesquelles les satellites correspondant aux différentes configurations atomiques ne sont pas ou très mal résolus; or, dans de tels cas, le désordre chimique est tout à fait comparable à celui présent dans les alliages qui sont, de ce fait, d'excellentes références. Pour des interfaces plus proches de la perfection, les satellites sont mieux résolus et une préconnaissance grossière de leur position relative est suffisante pour les identifier à une configuration chimique donnée. Dans le cas d'une interface parfaite, c'est à dire celui où la comparaison avec des alliages ou composés massifs est la plus discutable, le spectre ne présente qu'un satellite unique et bien résolu dont l'identification ne nécessite aucune référence.

\subsection{Analyse rapide des spectres de multicouches}

La première étape de l'analyse quantitative des spectres est faite en reproduisant, par ajustement, le spectre expérimental avec un jeu de raies gaussiennes centrées sur les fréquences déduites des études de référence (avec un peu de souplesse pour les raies résolues qui, comme vu plus haut, peuvent être légèrement déplacées). Des intensités relatives de ces raies on peut alors estimer la quantité de noyaux résonants dans telle ou telle gamme spectrale et donc les proportions d'atomes appartenant à telle ou telle région de l'échantillon, les deux principales régions étant les interfaces et le centre des couches. (voir le cas d'une multicouche $\mathrm{Co} / \mathrm{Cu}$, Fig.3). Il peut se faire que la partie du spectre correspondant aux interfaces soit tronquée du fait des limites instrumentales; dans ce cas la fraction d'atomes situés dans les interfaces peut être déduite de l'évolution de l'intensité de la partie massive du spectre avec l'épaisseur des couches. Pour des interfaces parfaites, la valeur trouvée pour la fraction d'atomes interfaciaux doit être de 1 monocouche par interface; la valeur, généralement plus grande, trouvée dans les multicouches réelles est une mesure quantitative immédiate du degré de mélange aux interfaces. Dans les systèmes granulaires (couches discontinues) le rapport d'intensité entre raies massives et raies d'interface est proportionnel au rapport volume/surface des grains; il est donc utilisé pour estimer la taille moyenne des grains ou leur forme [6,7]. Les proportions des différentes phases et autres surfaces internes (fautes d'empilement, joints de grains) dans la partie massive des couches sont aussi déterminées de cette façon, sachant qu'à chaque objet structural correspond une zone spectrale spécifique. Cependant les zones spectrales correspondant à des défauts massifs sont souvent partagées avec le spec-

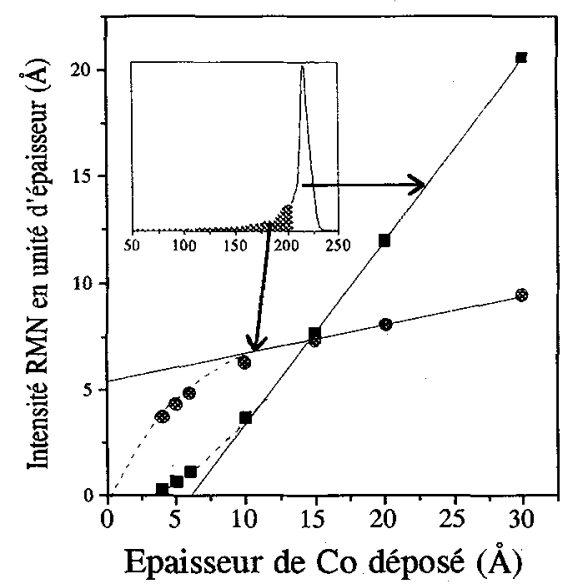

Fig.3. Variation avec l'épaisseur de Co déposé des intensités relatives de la partie interfaciale (cercles) et de la partie massive (carrés). La légère pente de l'intensité interfaciale provient des joints de grains dans la masse, son ordonnée à l'origine est la quantité de Co mélangé à l'interface. L'abscisse à l'origine de l'intensité massive mesure aussi la quantité de Co dans l'interface tre d'interface, il est donc essentiel d'effectuer des observations en fonction de l'épaisseur des couches pour discriminer la part interfaciale (indépendante de l'épaisseur) de la part massive (proportionnelle à l'épaisseur).

\subsection{Modèles d'interfaces}

Il est rare d'observer des interfaces parfaites et, généralement, les spectres d'interface s'étendent sur une gamme spectrale correspondant à plusieurs configurations atomiques. Dans de tels cas la modélisation de la forme des spectres permet d'obtenir des informations quantitatives détaillées sur la façon dont le mélange atomique se présente aux interfaces (rugosité à courte distance). Les spectres simulés consistent en une somme de $L$ raies primaires $(L=13$ dans les structures compactes) provenant des 
noyaux entourés de $\mathrm{L}-\mathrm{n}$ atomes du même élément et $\mathrm{n}$ atomes de l'autre élément. La distance entre les $\mathrm{L}$ raies est déterminée, en première approche, par les références, mais, comme discuté plus haut, un déplacement limité par rapport aux références est toujours permis pour tenir compte d'éventuelles différences entre la structure électronique aux interfaces et celle des références. Les raies sont supposées gaussiennes. La largeur des raies est un paramètre libre secondaire dans la procédure d'ajustement des spectres simulés aux spectres expérimentaux.

S'il est toujours facile de simuler les spectres expérimentaux avec un jeu de raies indépendantes, le jeu de probabilités de configuration résultant de cet ajustement (probabilités pour l'élément observé d'avoir $0,1,2, \ldots$ voisins étrangers à l'interface) $n$ 'apporte pas une information très parlante. C'est pourquoi il est préférable de considérer des modèles de structure d'interface (topologie et ordre chimique) dans lesquels les paramètres de base sont des distances, des tailles et des concentrations. De ces modèles on déduit les probabilités de configuration qui sont ensuite utilisées pour reproduire les spectres expérimentaux. La procédure d'ajustement consiste donc à raffiner des paramètres de nature structurale. Elle peut conclure à la non compatibilité ou à la compatibilité d'un modèle avec l'expérience et, dans ce dernier cas, elle fournit les valeurs de paramètres directement parlant, liés à l'ordre à courte distance aux interfaces. Les deux modèles de base qui peuvent être utilisés (éventuellement en combinaison ou variante) pour l'analyse des spectres interfaciaux sont présentés ci-dessous (Fig.4).

Quand l'analyse rapide suggère que les interfaces sont abruptes (faible mélange entre les deux éléments), le premier modèle d'interface auquel on pense est une interface dont les défauts sont essentiellement des marches monoatomiques $[5,8]$. Des variantes de ce modèle peuvent être imaginées où la seule couche atomique mixte est constituée d'îlots de l'un des éléments entourés de l'autre élément. Le modèle étant donné, les probabilités d'occurrence des différentes configurations de voisins ne dépendent que de quelques longueurs caractéristiques, distance moyenne entre marches ou diamètre moyen d'îlots. Ces longueurs sont les paramètres principaux qui, si le modèle s'avère applicable, caractérisent la nanostructure des interfaces. Dans les illustrations ci-dessous (Fig.4.a) deux paramètres suffisent pour décrire, dans le plan mixte, la densité des marche (d: distance moyenne) et leur géométrie (l: longueur moyenne des parties rectilignes). Il est important de remarquer que la RMN donne une information essentiellement sur les distributions de configuration des proches voisins et qu'en conséquence la rugosité ou le mélange atomique est caractérisé à l'échelle de 2 distances atomiques. Ainsi ce qu'on appelle ici interface abrupte peut aussi bien être une interface oblique ou ondulée pour autant que la pente soit sensiblement inférieure à $1 / 2$ : pour la RMN de telles interfaces (rugueuses à longue distance) apparaîtront abruptes, épaisses d'une monocouche, alors que les profils de densité électronique obtenus par rayons X apparaîtront plus épais. Ceci montre la complémentarité des mesures RMN et des mesures structurales standards pour distinguer entre un mélange intime et une rugosité plus étendue.
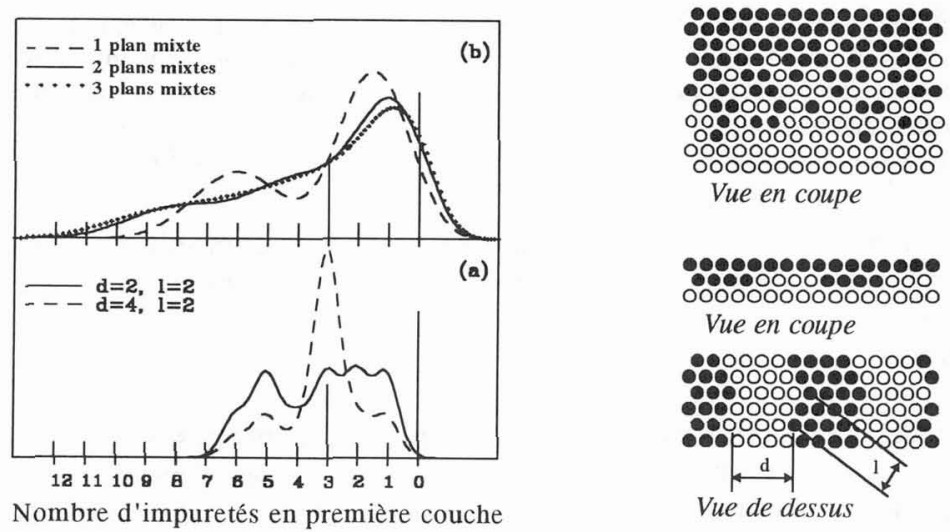

Fig.4. Modèles de base d'interfaces enfouies et les spectres RMN correspondants: (a) modèle à marches monoatomiques (d et l en unités de distance atomique); (b) modele d'interface diffuse avec profil de concentration linéaire. Les spectres simulés sont normalisés à une aire unité. 
Ces modèles de défauts en marches monoatomiques sont valables dans des situations où les deux éléments s'agglomèrent par espèce dans le plan mixte (cas de faible affinité chimique). Des déviations deviennent vite évidentes sur les spectres simulés dès qu'il existe une probabilité non négligeable pour que les atomes des deux espèces se dispersent mutuellement. Ces derniers cas sont beaucoup mieux interprétés selon un modèle d'interface diffuse (même avec un seul plan mixte) tel que présenté cidessous.

Quand, à l'évidence, il y a beaucoup plus qu'un plan atomique mélangé aux interfaces, on peut utiliser un modèle général décrivant le profil de concentration sur plusieurs plans [8,9]. On suppose alors que ces deux éléments sont distribués aléatoirement dans chaque plan (c'est à dire que l'interface diffuse est construite par une succession de solutions solides à deux dimensions). La distribution binomiale 2D pour chaque plan atomique définit les probabilités de configuration et la forme du spectre est totalement déterminée par la composition chimique des plans successifs. Les concentrations dans chaque plan sont les paramètres libres du modèle et, après ajustement au spectre expérimental, fournissent le profil de concentration avec une résolution atomique. De l'ajustement on déduit également le profil de champ hyperfin qui reflète approximativement le profil d'aimantation aux interfaces ( $\mathrm{Cf}$. 3.2). Le nombre de paramètres libres (1 par plan mixte) peut être réduit en contraignant le profil de concentration à suivre une loi analytique. Ainsi un profil linéaire n'a qu'un paramètre ajustable: sa pente ou, réciproquement le nombre de plans mixtes (Fig.4.b). Cependant, dans ce cas, la forme choisie pour le profil peut être incompatible avec l'expérience et sera rejetée par le test d'ajustement. Bien sûr, dans le monde réel, la distribution de configurations atomiques peut être différente d'une distribution binomiale mais il est difficile, dans la pratique, de développer un modèle qui tiendrait compte, à la fois, d'un profil de concentration selon la direction de dépôt et de l'ordre à courte distance détaillé dans le plan de dépôt. De telles tentatives sont décrites plus loin. La figure 4 présente les formes de spectres interfaciaux simulés selon les deux modèles de base pour plusieurs valeurs de leur paramètres pertinents [8]. On peut voir que ces formes sont très différentes entre ces deux modèles, même dans le cas commun d'un seul plan mixte, ce qui montre que les deux types de structure d'interface peuvent être aisément distingués. Dans le cadre du modèle d'interface diffuse, les différences de forme sont visibles jusqu'à trois couches mélangées. Au delà, la seule forme ne suffit pas pour prédire l'épaisseur de la couche mélangée mais, bien sûr, cette information est contenue quantitativement dans le rapport de l'intensité du spectre interfacial à celle du spectre total connaissant, ce qui est généralement le cas, l'épaisseur totale déposée (analyse rapide). Des exemples d'ajustement de spectres modèles aux spectres expérimentaux sont donnés dans la seconde partie de cet article.

Les deux modèles de base sont incapables de décrire les spectres observés lorsque le mélange aux interfaces est inhomogène, ainsi certaines multicouches dans lesquelles les interfaces sont, par endroits abruptes et, à d'autres, largement diffuses. Ce dernier cas est reconnaissable au fait que les spectres d'interfaces présentent à la fois une queue étendue peu structurée telles les interfaces diffuses et un satellite d'interface parfaite résolu. Ces spectres peuvent être reproduits dans un modèle où des îlots d'éléments purs sont dispersés dans une mer d'alliage. Les îlots sont supposés avoir une interface parfaite avec l'autre élément tandis que les parties alliées sont traitées comme un alliage homogène ou avec un profil de concentration comme dans le modèle d'interface diffuse. Ce modèle combine donc des version simplifiées des deux modèles de base avec pour paramètre supplémentaire le rapport d'aire entre les parties pures et les parties alliées dans le plan de croissance (Cf. 3.3).

\subsection{Procédure Expérimentale}

Du fait que l'analyse des spectres RMN en termes de structure est fondée sur les intensités relatives des différentes raies ou zones spectrales, il est nécessaire de discuter ici la question de l'intensité du signal RMN observé dans les matériaux ferromagnétiques. On sait que, dans les systèmes ferromagnétiques, les noyaux ne sont pas directement excités par le champ radiofréquence externe B1 mais au travers des oscillations du champ interne dues à la réponse des moments électroniques au champ externe. Ce fait donne lieu à une augmentation $\eta$ du champ radiofréquence effectif qui est proportionnelle à la perméabilité magnétique à la fréquence considérée. La valeur optimale du champ d'excitation externe -celle 
qui donne le signal RMN maximum- est donc $1 / \eta$ fois plus petite que la valeur nécessaire dans un système non magnétique. Réciproquement le signal observé ne provient pas directement des noyaux en précession mais des oscillations des moments électroniques induites par la précession nucléaire. Le signal RMN est ainsi également amplifié par le même facteur $\eta$. Ce fait présente le grand avantage d'une augmentation considérable de sensibilité puisqu'on peut détecter une monocouche de cobalt mais, si l'on ne tient pas compte de cet effet, l'intensité relative des raies RMN dans un ferromagnétique peut ne pas être une mesure exacte du nombre relatif de noyaux observés dans ces raies. Ceci est particulièrement vrai dans les échantillons inhomogènes parce que la perméabilité est généralement dépendante de la structure locale. En pratique, pourvu que le coefficient d'augmentation soit mesuré à toutes les fréquences par quelque moyen, ce problème est résolu. Le moyen le plus efficace consiste à enregistrer les spectres pour plusieurs amplitudes du champ radiofréquence sur une plage d'un ordre de grandeur autour de la valeur optimale. Les

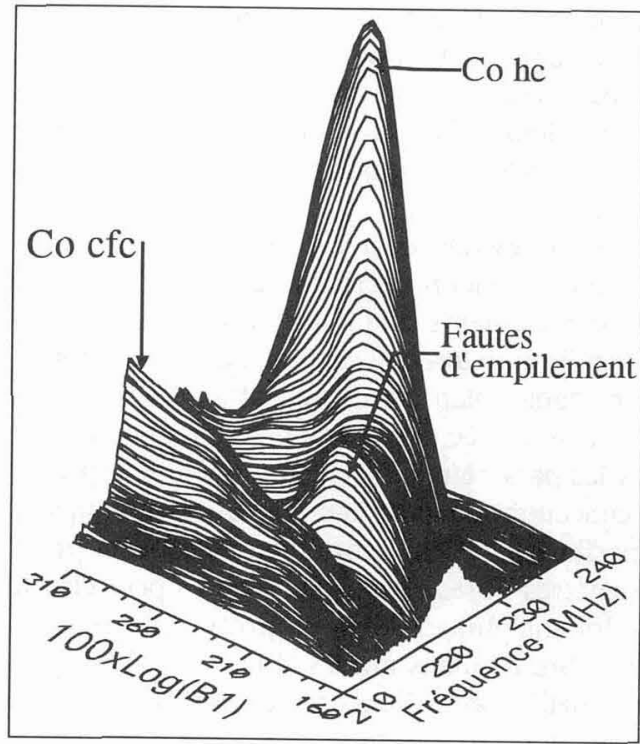

Fig.5. Intensité du signal RMN en fonction de la fréquence (spectre) et du champ d'excitation (B1 en échelle log.) telle qu'observée dans un'monocristal de Co hc (ou supposé tel).

données brutes obtenues (Signal RMN fonction de la Fréquence et du champ B1) donnent une figure $3 \mathrm{D}$ comme celle présentée sur la figure 5. De ces données l'amplitude relative exacte pour chaque fréquence est obtenue en utilisant la valeur au maximum en fonction du champ, multipliée par la valeur du champ pour ce maximum qui est précisément proportionnelle à $1 / \eta$. Tous les spectres présentés ici ont été obtenus par cette procédure dont la validité a été vérifiée dans les bonnes solutions solides (on obtient bien la distribution binomiale attendue pour les différentes configurations de voisins).

La procédure a un autre avantage puisqu'en sous-produit elle fournit la dépendance en fréquence de la perméabilité magnétique locale ou, plus exactement, la rigidité locale des moments magnétiques électroniques. Sachant qu'à chaque zone spectrale est associée une région spécifique dans un échantillon inhomogène, les courbes $S(F, B 1)$ telles que celle de la figure 5 fournissent une vue directe des inhomogénéités structurales et magnétiques d'un échantillon. A plus haut niveau, une telle étude permet de savoir quels paramètres structuraux, à l'échelle atomique, gouvernent le caractère dur ou doux des couches magnétiques (Cf. 3.1).

\section{EXEMPLES EXPERIMENTAUX}

\subsection{Eléments Non Miscibles: multicouches et sandwiches $\mathrm{Co} / \mathrm{Cu}$}

Dans cette sous-section nous résumons les études de la structure atomique des couches de Co dans les multicouches et sandwiches $\mathrm{Co} / \mathrm{Cu}$ d'origines variées. Différentes techniques ont été employées pour préparer ces multicouches (Epitaxie par Jet Moléculaire - EJM- ou bombardement électronique, pulvérisation cathodique continue ou haute fréquence) avec pour résultat des spectres RMN très différents. Ces différences proviennent de trois classes de différences structurales entre les échantillons: un mélange de phases cfc et hc pour le cobalt, une croissance colonnaire avec des grains de diamètres différents, et un mélange $\mathrm{Co}-\mathrm{Cu}$ à des degrés divers aux interfaces. La structure dans la masse des couches 
(mélange cfc-hc, fautes d'empilement et autres défauts) ainsi que la topologie des interfaces ont été étudiées en détail $[5,8,10-12]$.

Sur la figure 6 on peut voir une série typique de spectres observés dans les multicouches. Tous les spectres sont normalisés à l'aire totale d'interface de sorte que, si la structure et la composition des interfaces ne varie pas d'un échantillon à l'autre, leurs composantes spectrales sont superposées alors que les contributions du centre des couches croissent proportionnellement à l'épaisseur du cobalt. Les spectres peuvent donc, à première vue, être décomposés en deux parties: une structure principale entre $215 \mathrm{MHz}$ et $230 \mathrm{MHz}$ correspondant à la partie massive (Co cfc à $217 \mathrm{MHz}$, Co hc à $225-228 \mathrm{MHz}^{1}$, fautes d'empilement entre les deux) et un jeu de raies satellites ou une queue en dessous de $205 \mathrm{MHz}$ correspondant aux noyaux placés aux interfaces (où le $\mathrm{Co}$ a des $\mathrm{Cu} \mathrm{PV}$ ). Dans l'exemple choisi, les interfaces sont loin d'être parfaites et l'on peut tirer quelque information directe de la simple observation des spectres.

Ces spectres se superposent dans les basses fréquences ce qui montre que la topologie et le mélange interfacial ne dépendent pas de l'épaisseur de Co. On observe souvent cependant que, dans une série d'échantillons, les plus minces présentent des spectres interfaciaux différents des échantillons plus épais. Ici l'effet est visible mais faible. On peut l'expliquer parfois par l'existence de contraintes liées à la différence de paramètre de réseau entre les deux éléments [5]. Toutefois la cause la plus évidente du changement de forme du spectre interfacial se trouve liée au fait que les interfaces rugueuses s'interpénètrent lorsque la

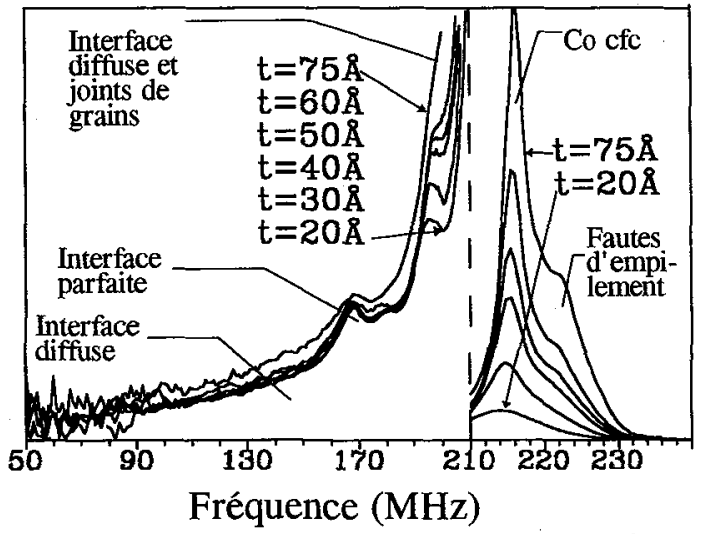

Fig.6. Exemples de spectre RMN du Co dans une série de vannes de spin $\mathrm{Cu} / \mathrm{Co}_{1} / \mathrm{Cu} / \mathrm{NiFe} / \mathrm{FeMn}$. A gauche: spectre interfacial (Agrandi 20 fois). A droite: spectre de la partie massive. couche devient plus mince que 2 fois l'amplitude des corrugations: l'absence de plan complet dans la couche (qui devient discontinue) résulte en une modification rapide des probabilités de configuration dans la totalité de la couche de Co et donc de tout le spectre RMN.

Les spectres donnés en exemple présentent un satellite bien visible à $165 \mathrm{MHz}$, qui correspond aux atomes de Co ayant $3 \mathrm{Cu}$ PV dans des zones d'interface (111) parfaite. Cependant l'intensité de ce satellite est faible au regard de la queue étendue observé à basse fréquence qui, elle, est caractéristique d'une interface diffuse. On peut donc conclure qualitativement au vu des spectres que les interfaces se composent de zones largement mélangées entourant des plages, en faible nombre, où la transition CoCu est abrupte.

On peut également voir sur la figure 6. une augmentation significative d'intensité dans la plage 200$210 \mathrm{MHz}$ lorsque l'épaisseur de Co augmente. Bien que cette zone spectrale corresponde aussi à des atomes d'interface (Co avec $1 \mathrm{Cu} \mathrm{PV}$ ), l'intensité croît linéairement en fonction de l'épaisseur déposée cf. Fig.3). Pour expliquer cette observation il est donc nécessaire de considérer une contribution d'atomes de Co dans la partie centrale des couches. Comme la zone de fréquence considérée est loin des fréquences de résonance du Co massif ( $\mathrm{cfc}$ ou hc) le signal observé dans cette plage doit être attribué à des défauts dans la masse. L'analyse chimique des échantillons ayant exclu la présence d'impuretés, il en a été conclu que ce signal provenait d'atomes de Co aux joints des nombreux grains colonnaires dont sont formées ces multicouches. La proportion de ces atomes par rapport à la quantité de Co déposée représente le rapport surface/volume des grains et, en supposant que les colonnes sont hexagonales, l'analyse quantitative a permis d'estimer leur diamètre moyen à $60 \AA$. Cette valeur a été confirmée par la suite grâce à des images de coupes par microscopie électronique à transmission. Par con-

\footnotetext{
' Dans le cobalt hc le champ hyperfin est anisotrope: lorsque l'aimantation est alignée avec l'axe c la fréquence RMN est 220-222 MHz, dans le plan elle vaut 225-228 MHz. Pour tous les exemples donnés dans l'article l'aimantation est dans le plan dense $(111 \mathrm{c})$ ou $(001 \mathrm{~b})$.
} 
traste, dans d'autres séries d'échantillons formés de gros grains (>300§) on n'observe pas de variation décelable d'intensité dans cette région spectrale.

La dernière observation qualitative que l'on peut faire sur ces spectres concerne la structure cristallographique des couches de Co. La raie étroite autour de $217 \mathrm{MHz}$ comparée à la faible intensité autour de $228 \mathrm{MHz}$ montre que le Co est avant tout cfc mais l'intensité considérable entre ces deux fréquences indique la présence d'un nombre important de fautes d'empilement. De plus la quantité relative de Co dans des environnements hc (contribution à haute fréquence) augmente avec l'épaisseur de Co. On en conclut que le cobalt croît d'abord cfe sur le cuivre puis avec l'augmentation d'épaisseur tend à recouvrer sa structure stable hc par un nombre croissant de fautes d'empilement.

En ce qui concerne la structure des couches de Co dans la masse les études effectuées peuvent se résumer ainsi: (I) le cuivre favorise une croissance cfc du cobalt, sur une couche (111) de $\mathrm{Cu}$ le cobalt croit initialement avec un empilement cfc et, à épaisseur constante de $\mathrm{Co}$, plus la couche de Cu est épaisse plus la proportion de Co cfc est grande (quelques exceptions de croissance he du Co sur Cu ont toutefois été observées [13]); au contraire, (II) les fortes épaisseurs de Co et une texture (111) très marquée favorisent la structure hc plus stable; (III) les forts taux de déposition favorisent la présence de nombreux grains colonnaires de petit diamètre.

Les résultats de l'étude de la structure des interfaces dans ces multicouches sont résumés sur la figure 8. On peut y voir que les échantillons présentent de très grandes différences qualitatives. Ces différences peuvent être traduites quantitativement en termes de rugosité/mélange aux interfaces par comparaison avec des spectres simulés selon les modèles présentés plus haut (Fig.4). Les exemples sont présentés, de haut en bas, par ordre croissant de rugosité d'interface (ou de mélange $\mathrm{Co}-\mathrm{Cu}$ ).

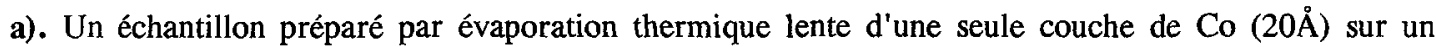
monocristal de cuivre (111). La couche de Co a été recouverte d'une couche de fer. Le but de cette préparation était d'obtenir une seule interface $\mathrm{Co} / \mathrm{Cu}$ la plus parfaite possible. Le choix du fer comme couche finale était dicté par le fait que l'interface $\mathrm{Fe} / \mathrm{Co}$, non nécessairement abrupte, produit des satellites à plus haute fréquence que le Co pur et donc sans recouvrement avec l'interface $\mathrm{Co} / \mathrm{Cu}$. Le spectre attendu pour une couche avec une interface $\mathrm{Co} / \mathrm{Cu}$ parfaite est censé consister en deux raies étroites, une pour les noyaux au centre de la couche (à la fréquence du Co pur), une pour les atomes de Co à l'interface $\mathrm{Co} / \mathrm{Cu}$ qui ont $3 \mathrm{Cu}$ voisins pour la direction de croissance considérée. De fait le spectre expérimental est très proche de celui attendu théoriquement, à la fois qualitativement (on n'observe pratiquement qu'un seul satellite à basse fréquence de largeur comparable à la raie principale) et quantitativement (l'intensité de ce satellite correspond presque exactement à une monocouche de $\mathrm{Co}$ ). La fréquence du satellite est ici environ $5 \%$ plus basse que celle attendue pour $3 \mathrm{Cu} \mathrm{PV}$ d'après les études d'alliages massifs: ceci illustre les modifications du champ hyperfin qui peuvent être dues aux contraintes et/ou à l'anisotropie d'environnement chimique à l'interface parfaite. Dans les autres échantillons, où le désordre chimique est plus important à l'interface, la position du satellite est beaucoup plus proche, comme prévu, de la position observée dans les alliages. Le satellite à haute fréquence appartient à l'interface $\mathrm{Fe} / \mathrm{Co}$ (cf. 3.3).

b). Un échantillon en multicouches préparé par évaporation thermique lente sur un tampon d'or lui-même déposé sur float-glass. Bien que l'échantillon soit polycristallin dans le plan de croissance, la couche tampon d'or présente une surface très plane, de très grands grains et elle favorise une excellente texture (111) dans la di-

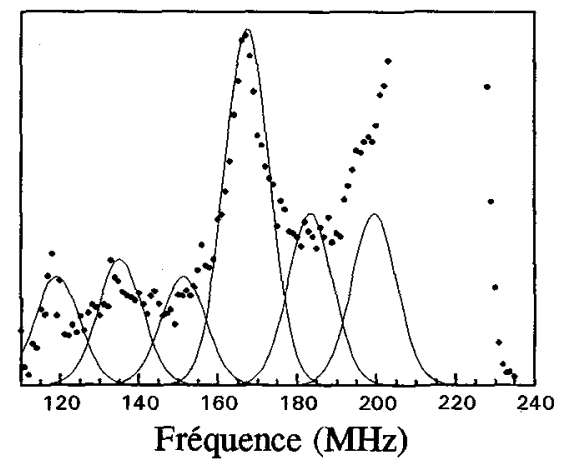

Fig.7. Reconstruction d'un spectre de multicouche $\mathrm{Co} / \mathrm{Cu}$ montrant les contributions de configurations avec 1 à $6 \mathrm{Cu}$ voisins à l'interface: modèle d'interface à marches monoatomiques $(\mathrm{d}=2,5,1=1,6)$. L'intensité supplémentaire autour de $200 \mathrm{MHz}$ est due aux joints de grains. Les contributions du Co pur ne sont pas représentees. 
rection de croissance. Le spectre présente, comme dans le cas précédent, un satellite dominant caractéristique d'interfaces abruptes. L'intensité totale du spectre interfacial contient l'équivalent de 1,1 monocouche de Co ce qui montre que le mélange interfacial est très faible. Les défauts aux interfaces peuvent être décrits par des marches monoatomiques séparées, en moyenne, de 4 distances atomiques $(75 \%$ d'aire d'interface parfaite). La figure 7 montre un autre exemple de spectre pour un échantillon de qualité équivalente, produit, celui-là, par EJM ainsi que la reconstruction de ce spectre selon le modèle à marches monoatomiques.

c,d,e). Echantillons obtenus par pulvérisation cathodique sur des substrats $\mathrm{Si}$ avec différentes couches tampons et destinés, plus spécialement, aux études de magnétorésistance. Ils présentent à des degrés divers un mélange $\mathrm{Co}-\mathrm{Cu}$ aux interfaces nettement plus important que les précédents les interfaces contenant l'équivalent de 1.5 monocouche de Co pur (interfaces encore assez abruptes mais avec une forte densité de marches atomiques) à 3 monocouches de Co pur (environ 6 plans atomiques ou $12 \AA$ contenant du Co et du $\mathrm{Cu}$ ). Des séries assez grandes de ces échantillons (5 à 10) avec différentes épaisseurs de $\mathrm{Co}$ ou de $\mathrm{Cu}$ et différentes périodes ont permis d'étudier l'influence de divers paramètres sur la nanostructure des interfaces: (I) Les forts taux de dépôt (magnétron plutôt que diode) favorisent la production d'interfaces abruptes (spectre typique c) alors que (II) à faible taux de dépôt, la croissance en colonnes de plus grand diamètre provoque la formation d'une forte rugosité à longue distance (vue par microscopie à force atomique), une faible texture de croissance (diffraction $\mathrm{X}$ ) et, à courte distance (RMN), une absence totale d'interface abrupte mais au contraire, un très fort mélange à l'échelle atomique (spectre typique e); (III) la nature de la couche tampon entre le substrat et la multicouche a un effet dramatique sur la qualité des interfaces puisque des échantillons préparés sur tampon de $\mathrm{Cu}$, toutes choses égales par ailleurs, présentent un spectre caractéristique d'interfaces désordonnées et diffuses alors que ceux préparés sur tampon de Fe ont un spectre caractéristique d'interfaces abruptes à marches monoatomiques. Le spectre (d) appartient à la famille d'échantillons ayant des îlots d'interfaces abruptes dans une mer d'alliage $\mathrm{Co}-\mathrm{Cu}$.

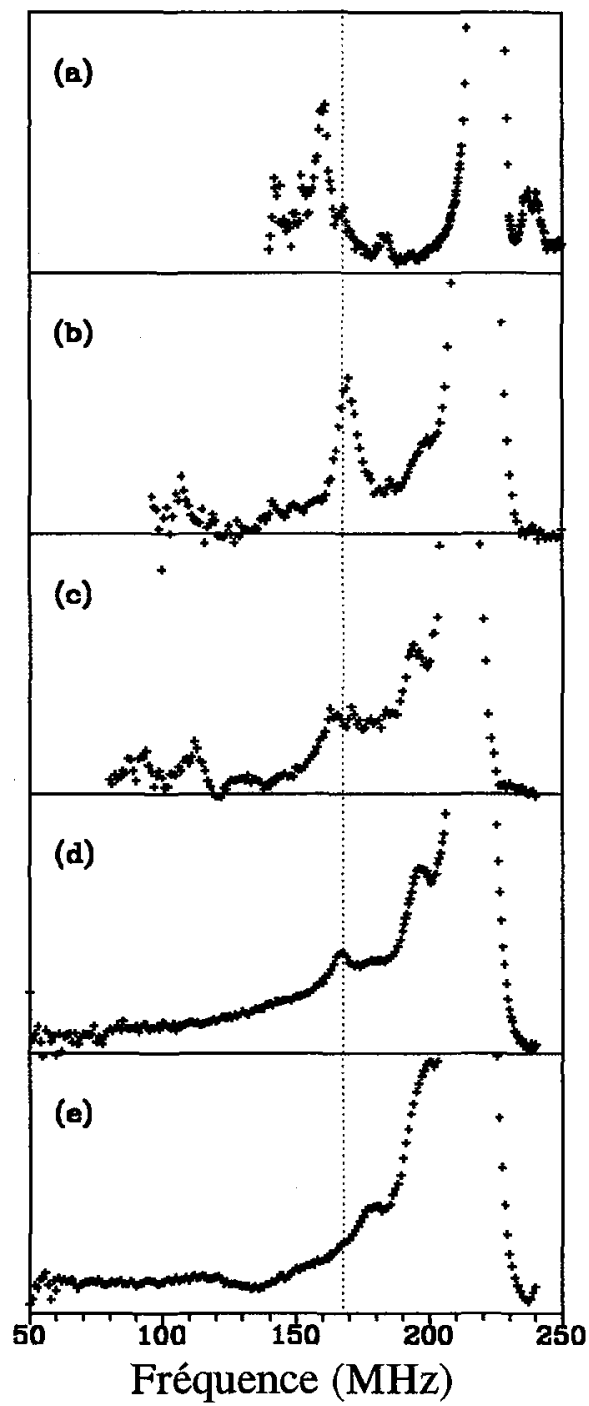

Fig.8. Exemples de spectres d'interfaces ... $/ \mathrm{Cu} / \mathrm{Co} /$. . . Le trait pointillé vertical indique la position du satellite de Co à $3 \mathrm{Cu}$ Premiers Voisins dans les alliages massifs.

a) Interface (111) presque parfaite (1 monocouche). Un seul satellite correspondant au $\mathrm{Co}$ avec $3 \mathrm{Cu}$ PV. Traces de Co avec $2 \mathrm{Cu}$ PV.

b) La meilleure' multicouche observée $(1,1 \mathrm{MC}$ à l'interface). La raie à $3 \mathrm{Cu} \mathrm{PV}$ contient $30 \%$ du total de l'interface. La distance moyenne entre marches monoatomiques est de 4 rayons atomiques.

c) Interface abrupte $(<1,5 \mathrm{MC})$ mais avec de nombreuses marches (distance moyenne: 2 rayons atomiques).

d) Interface mixte (2,5 MC). Présence de zones plates (raie à $3 \mathrm{Cu}$ PV résolue). Profil de concentration étroit.

e) Interface diffuse (>3 MC.). L'intensité des satellites à 1 et $2 \mathrm{Cu} \mathrm{PV}$ inđique la présence đe plusieurs plans contenant une faible concentration de $\mathrm{Cu}(3-8 \%)$. Satellite parfait (3 $\mathrm{Cu} \mathrm{PV}$ ) pratiquement inexistant. 


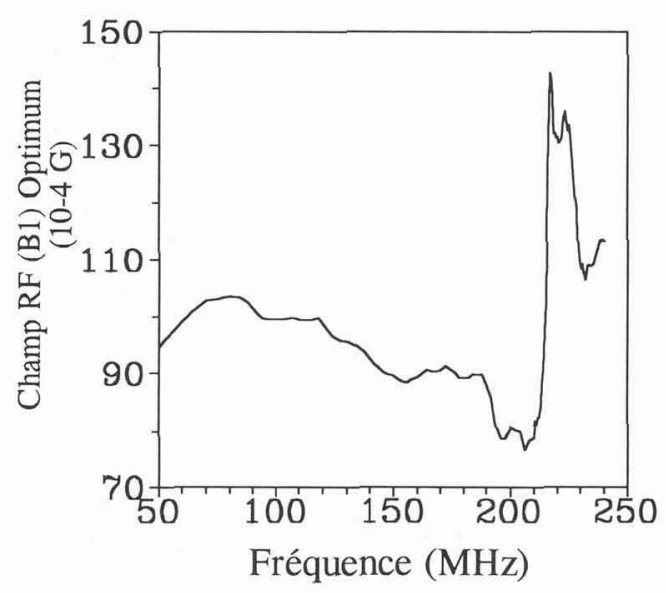

Fig.9. Variation du Champ B1 optimum en fonction de la fréquence (rigidité magnétique) dans une vanne de spin $\mathrm{Cu} / \mathrm{Co} / \mathrm{Cu} / \mathrm{NiFe} / \mathrm{FeMn}$ : douceur magnétique aux joints de grains $(200-210 \mathrm{MHz})$ et aux interfaces $(<200 \mathrm{MHz})$, rigidite au centre des couches $(>210 \mathrm{MHz})$.

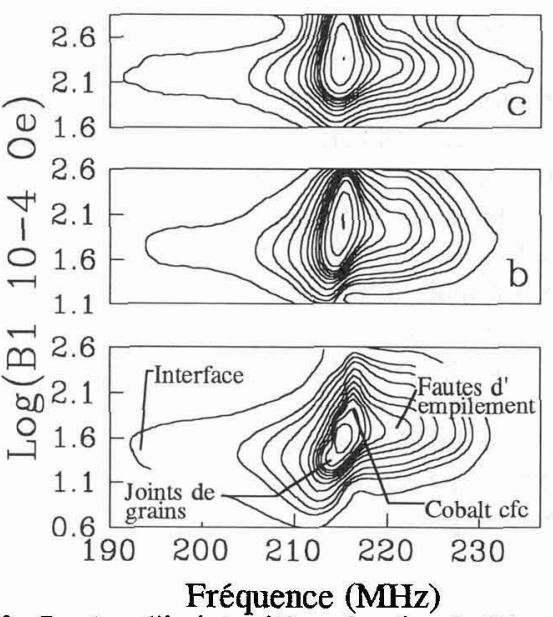

Fig.10. Courbes d'isointensité en fonction de fréquence et de $\mathrm{B} 1$ dans les vannes de spin $\mathrm{Cu} / \mathrm{Co} / \mathrm{Cu} / \mathrm{NiFe} / \mathrm{FeMn}$ (non traitées et recuites à b: $280^{\circ} \mathrm{C}, \mathrm{c}: 320^{\circ} \mathrm{C}$ ). On voit le durcissement des couches (augmentation du B1 optimum) avec le recuit du à la disparition des joints de grains doux.

L'applicabilité des multicouches $\mathrm{Co} / \mathrm{Cu}$ en tant que capteurs magnétorésistifs dépendant, de manière critique, de leur douceur/dureté magnétique, il est utile de discuter sur cet exemple de l'apport très spécifique de la RMN sur ce point. Comme indiqué dans la section II, l'analyse des données brutes permet de mesurer la rigidité relative des moments magnétiques dans les différentes parties d'un échantillon inhomogène (ce qui est le cas, par essence, d'une multicouche et, a fortiori, si elle présente des défauts structuraux).

L'étude rapportée ici a ainsi montré que les différentes régions de couches magnétiques de Co peuvent être classées par ordre de rigidité magnétique croissante (pour des moments tournants dans le plan dense (111)) selon : 1) les joints de grains, 2) les interfaces, 3) les fautes d'empilement hc, 4) la phase massive cfc. Ceci est illustré sur la figure 9 qui montre la variation de l'amplitude optimale du champ B1 en fonction de sa fréquence: les moments sur les surfaces internes telles les interfaces (basses fréquences) et surtout les joints de grains $(200-210 \mathrm{MHz})$ ont clairement besoin d'un plus faible B1 pour tourner que les moments dans le volume des couches $(215-230 \mathrm{MHz})$. Selon que l'on veut que la couche de Co soit dure ou douce on devra donc choisir les conditions de préparation (comme vu plus haut) qui favorisent les régions adéquates. Après recuit thermique des échantillons les mesures macroscopiques (cycles d'aimantation, magnétorésistance) montrent un durcissement magnétique des couches initialement douces. La figure 10 , qui présente les courbes d'isointensité $R M N S(F, B 1)$, illustre la raison de ce durcissement: en effet, on peut y voir la disparition progressive des joints de grains doux (les grains grandissent et coalescent) associée au durcissement considérable de l'ensemble de la couche (le champ optimum moyen devient de plus en plus fort). Des mesures de microscopie électronique ont confirmé par la suite la croissance des grains après recuit.

\subsection{Eléments miscibles: multicouches $\mathrm{Co} / \mathrm{Cr}, \mathrm{Co} / \mathrm{Ru}$}

Le chrome et le ruthénium sont des éléments qui, mélangés au cobalt, forment une solution solide (bien que l'on soupçonne la possibilité d'une lacune de miscibilité dans le diagramme de phase Co-Cr). Dilués dans le Co, les deux éléments stabilisent la phase hc du Co. Ru est hexagonal lui-même et dans les super-réseaux $\mathrm{Co} / \mathrm{Ru}$ l'empilement est hc. Dans les super-réseaux $\mathrm{Co} / \mathrm{Cr}$ les fortes épaisseurs de $\mathrm{Cr}$ tendent à imposer la structure $\mathrm{cc}$ au Co, mais à faible épaisseur le cobalt reste hc alors que le chrome crôit avec une structure cc distordue, selon plusieurs directions d'épitaxie (variants), de façon à 
s'adapter à la symétrie hexagonale des plans (001h) du Co hc [4]. Dans les multicouches Co/Ru, les effets des conditions de préparation sur la structure des interfaces se sont révélés bien moindres que dans le cas précédent du système $\mathrm{Co} / \mathrm{Cu}$ : les échantillons préparés par $\mathrm{EJM}$ ou pulvérisation, à des températures de substrat variant de $0^{\circ} \mathrm{C}$ à $150^{\circ} \mathrm{C}$, ne présentent que des différences subtiles sur les spectres RMN du Co. Ceci est à attribuer, à l'évidence, à l'affinité chimique des éléments qui tendent naturellement à s'allier contrairement au $\mathrm{Cu}$ et au Co.

Les études pour différentes conditions de préparation du système $\mathrm{Co} / \mathrm{Cr}$ ont été moins poussées et limitées au cas de faibles épaisseurs de Cr. La grande similitude des spectres montre que les multicouches $\mathrm{Co} / \mathrm{Cr}$ comme $\mathrm{Co} / \mathrm{Ru}$ présentent des structures d'interfaces très semblables: ce sont deux cas où l'interdiffusion est forte, proche de celle des alliages massifs.

Les spectres typiques de trois échantillons $\mathrm{Co} / \mathrm{Cr}$ sont présentés sur la figure 11 . Bien que la diffraction de rayons $X$ montre que la structure de ces multicouches est globalement hc, la raie principale du spectre n'est qu'à $222 \mathrm{MHz}$ ce qui, pour une aimantation dans le plan de base hc, est faible (la fréquence attendue est de 225-228 MHz). La conclusion de cette observation, en accord avec la microscopie électronique, est que les échantillons présentent une forte densité de fautes d'empilement. Cette structure assez peu cohérente explique aussi la largeur de la raie principale comparée à la raie aiguë du Co cfc observée dans les échantillons $\mathrm{Co} / \mathrm{Cu}$. Le léger déplacement vers les basses fréquences dans l'échantillon ayant la plus mince couche de Co pourrait être expliquée par les contraintes d'interfaces mais, beaucoup plus vraisemblablement, il est dû à la moindre aiman-

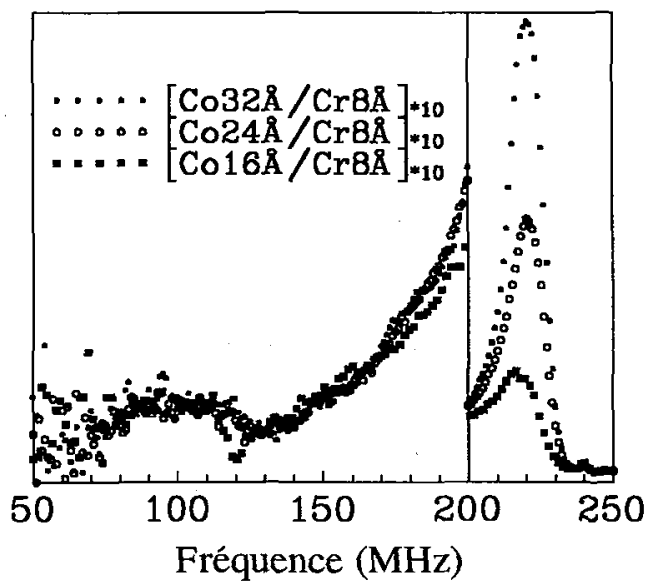

Fig.11. Spectres RMN du Co dans les multicouches $\mathrm{Co} / \mathrm{Cr}$. Spectre interfacial (50-200 MHz) agrandi 4 fois. tation moyenne de l'échantillon car la présence de chrome diminue très rapidement le moment du Co dans son voisinage (effet de la structure électronique moyenne). La longue queue des spectres en basse fréquence provient des atomes de Co les plus proches $\mathrm{du} \mathrm{Cr}, \mathrm{c}^{\prime}$ est à dire les atomes aux interfaces. Ces queues se superposent pour les trois épaisseurs de Co ce qui montre que la morphologie des interfaces ne dépend pas de l'épaisseur de Co. De la variation avec l'épaisseur de l'intensité relative de la raie principale (atomes de Co dans la masse des couches) la quantité de Co présente dans les interfaces diffuses est estimée à $11 \AA$ par couche de Co; notons qu'ici on ne peut utiliser directement l'intensité relative de la partie interfaciale du spectre car les atomes de moment faible ne sont pas tous observés, le spectre est tronqué vers les basses fréquences. De fait dans les échantillons pour lesquels l'épaisseur de Co est inférieure à $10 \AA$ aucun signal RMN n'a pu être observé le cobalt étant allié à coeur et trop faiblement magnétique.

De tels cas de fort mélange interfacial sont interprétés, de manière très adéquate, dans le cadre du modèle d'interface diffuse. A première vue la forme des spectres d'interface correspond très bien à celle des spectres théoriques présentés dans la section 2 . Un exemple de reconstruction théorique d'un spectre expérimental est donné sur la figure 12; on peut y voir la contribution de chaque plan atomique composant l'interface épaisse. Cette reconstruction illustre une différence possible entre spectre d'interface et spectre d'alliage massif dans le cas d'une structure anisotrope. Dans les alliages massifs, les satellites correspondant aux configurations à $0,1,2, \ldots \mathrm{Cr}$ voisins sont espacés de $32 \mathrm{MHz}$ (cf. Fig.1). Les deux exemples d'ajustement théorique au spectre expérimental des multicouches montrent clairement qu'une valeur plus faible de cet espacement $(20 \mathrm{MHz})$ donne un meilleur accord que la valeur de $32 \mathrm{MHz}$. En fait, dans les alliages hexagonaux $\mathrm{Co}-\mathrm{Cr}$ dilués [14], on observe des satellites jumeaux, d'égale intensité, à $41 \mathrm{MHz}$ et $22 \mathrm{MHz}$ de la raie principale: la présence de deux satellites plutôt q'un seul pour la configuration la plus probable $(1 \mathrm{Cr}$ parmi les $12 \mathrm{PV})$ doit être associée avec l'équiprobabilité de trouver $1 \mathrm{Cr}$ parmi les $6 \mathrm{PV}$ dans le même plan $(001 \mathrm{~h})$ que le noyau Co sondé ou 1 parmi les 
$3+3$ PV dans les 2 plans adjacents. L'espacement de $32 \mathrm{MHz}$ dans les alliages concentrés est précisément la position moyenne de ces deux satellites lorsqu'ils s'élargissent et se recouvrent en présence d'un fort désordre chimique à longue distance.

Ce constat étant fait, le résultat observé dans les multicouches n'est pas surprenant puisque, contrairement aux alliages dans lesquels la composition chimique est isotrope, le gradient de concentration aux interfaces augmente significativement la probabilité de trouver des $\mathrm{Cr}$ voisins dans un plan adjacent (vers le centre de la couche de $\mathrm{Cr}$ ). La situation est strictement identique pour le $\mathrm{Co} / \mathrm{Ru}$. Incidemment cette remarque permet d'attribuer, parmi les deux satellites observés dans les alliages, celui à $22 \mathrm{MHz}$ aux impuretés de $\mathrm{Cr}$ hors du plan et celui à $41 \mathrm{MHz}$ aux impuretés dans le plan (001h) du noyau sondé. Le profil de concentration déduit des ajustements est présenté sur la figure 13 (la gamme de valeurs possibles correspond à des espacements entre satellites de $32 \mathrm{MHz}$ à $20 \mathrm{MHz}$ ). Ce profil montre que l'interface s'étend sur 5 plans par interface. Toutes les concentrations indiquées n'ont pas la même précision. En effet, comme vu sur la figure 12, le spectre, dans la gamme de fréquence explorée, comprend les contributions de trois plans seulement: de droite à gauche, le dernier plan complet de Co vu comme un épaulement de la raie principale, puis les deux premiers plans mixtes. Seules les concentration de ces deux plans sont donc directement mesurées. Le troisième plan mixte, trop peu magnétique pour contribuer au spectre, influence cependant la forme spectrale du plan précédent et sa concentration est donc obtenue indirectement. En revanche, les deux derniers plans mixtes ne sont, ni directement, ni indirectement observés mais leur composition est bornée pour satisfaire aux contraintes i) de la quantité totale de $\mathrm{Cr}$ déposée et ii) d'un profil monotone. Comme on peut le voir, ces contraintes imposent que les couches minces de $\mathrm{Cr}$ ne contiennent aucun plan pur en $\mathrm{Cr}$. De la fréquence moyenne de résonance dans chaque plan atomique il est aussi possible d'estimer le profil d'aimantation dans l'interface: bien que le champ hyperfin ne soit pas exactement proportionnel au moment local, sa valeur moyenne fournit une estimation raisonnable de la valeur relative du moment de chaque plan atomique. Le profil de champ hyperfin (d'aimantation relative) à l'interface $\mathrm{Co} / \mathrm{Cr}$ est présenté sur la figure 13; en raison de son caractère intégral, il est moins sensible au choix de l'espacement entre satellites que le profil de concentration. En intégrant le profil d'aimantation on estime la perte d'aimantation moyenne par interface $\mathrm{Co} / \mathrm{Cr}$ ( 1,8 couches mortes) qui se compare directement aux mesures d'aimantation macroscopique et $\mathbf{s}^{\prime}$ avère en excellent accord avec elles.

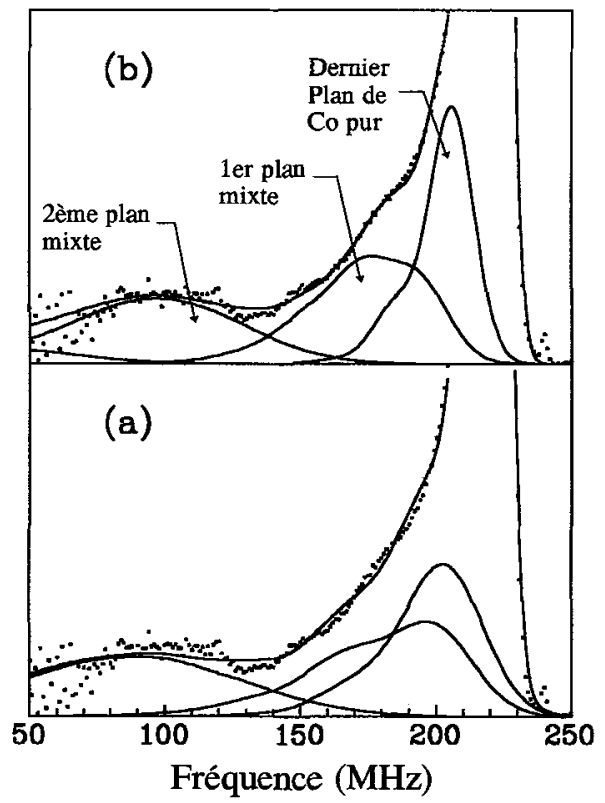

Fig.12. Reconstruction d'un spectre de multicouche $\mathrm{Co} / \mathrm{Cr}$ montrant la contribution des plans atomiques composant l'interface diffuse. a) avec la meilleure valeur d'espacement entre satellites (20 MHz); b) avec la valeur d'espacement trouvée dans les alliages de référence (32 $\mathrm{MHz}$ ). Contribution du centre des couches non représentée.

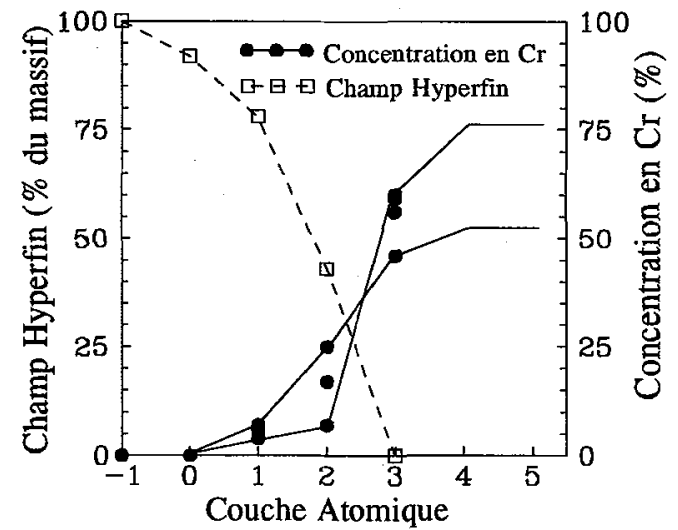

Fig.13. Profil de la concentration en $\mathrm{Cr}$ et du champ hyperfin (aimantation) au travers de l'interface diffuse $\mathrm{Co} / \mathrm{Cr}$. La couche atomique 0 est le dernier plan de Co complet. Les courbes pleines indiquent les valeurs extrêmes de concentrations compatibles avec l'expérience. 
La structure des multicouches $\mathrm{Co} / \mathrm{Ru}$, comme mentionné plus haut, est semblable à celle du système $\mathrm{Co} / \mathrm{Cr}$ aussi l'étude n'en sera-t-elle pas dêveloppée. Cependant ce système offre une autre occasion d'illustrer une mesure de rigidité magnétique par RMN. En effet dans les multicouches $\mathrm{Co} / \mathrm{Ru}$, les couches de Co sont couplées au travers du $\mathrm{Ru}$ et ce couplage magnétique oscille de ferromagnétique à antiferromagnétique avec une période d'environ $10 \AA$. Comme le couplage antiferromagnétique rend plus difficile la rotation des moments sous l'influence du champ B1, l'oscillation de couplage est aussi observée, comme le montre la figure 14 , sur la variation du B1 optimum (ici la moyenne sur tout le spectre) en fonction de l'épaisseur de $\mathrm{Ru}$.

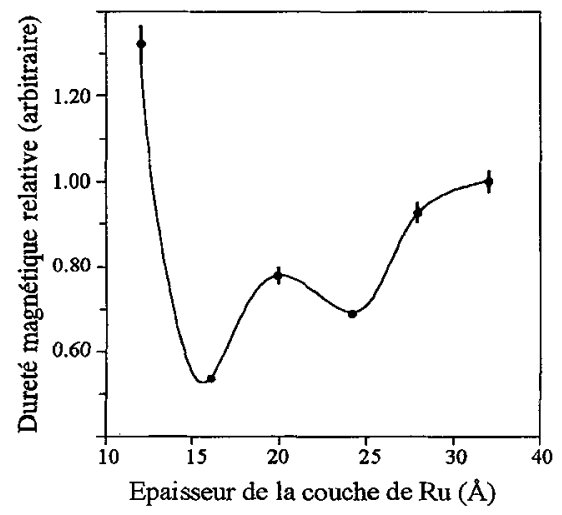

Fig.14. Oscillations de la rigidité magnétique des couches de Co dans les multicouches Co/Ru observées par RMN (B1 optimum). Elles sont dues aux oscillations de couplage entre couches de Co au travers du Ru.

\subsection{Eléments formant des composés intermétalliques: super-réseaux $\mathrm{Co} / \mathrm{Fe}$}

Le cobalt peut être stabilisé dans une structure cubique centrée par pseudomorphie avec le fer dans des multicouches $\mathrm{Co} / \mathrm{Fe}$ [15]. Pour des super-réseaux fabriqués par EJM, la diffraction $\mathrm{X}$ et les expériences de canalisation d'ions montrent la présence de la phase cubique centrée jusqu'à une épaisseur critique de $21 \AA$ de cobalt déposé, à l'exclusion de toute autre phase cfc ou hc. En revanche, durant la croissance de ces mêmes super-réseaux, les observations RHEED de la surface de Co libre montrent que cette surface adopte un empilement hc dès $10 \AA$ déposés seulement [16-18]. Ces résultats impliquent une transformation de la couche de Co sous-jacente lorsqu'on la recouvre de fer. Des expériences RMN ont donc été effectuées dans ces super-réseaux pour des épaisseurs de $5 \AA$ à $42 \AA$ de Co afin i) de comprendre les raisons de la contradiction apparente entre les mesures de diffraction de surface et de volume et ii) d'étudier la structure à courte distance aux interfaces $\mathrm{Co} / \mathrm{Fe}$. Nous ne présentons ici que l'étude d'une série d'échantillons déposés à $175^{\circ} \mathrm{C}$ sur substrat GaAs (110). Les effets de l'épaisseur de fer et l'influence du substrat et de son orientation $(\mathrm{MgO}, 100)$ ont également été étudiés. Les observations dans la série (110) sont résumées sur la figure 15 . Pour des épaisseurs de Co entre $5 \AA$ et $21 \AA ̊$ les spectres présentent, sauf à faible épaisseur, un pic principal à $198 \mathrm{MHz}$ et un satellite étroit et bien résolu à $214 \mathrm{MHz}$ puis une queue étendue vers les hautes fréquences. La raie à $198 \mathrm{MHz}$ est bien en dessous de toutes les fréquences connues du Co hc et cfc et doit être attribuée à la structure cc comme l'ont mis en évidence pour la première fois des mesures combinées d'EXAFS et de RMN [15]. Contrairement aux autres éléments, la proximité du fer augmente la fréquence de résonance du Co [2], il est donc difficile sur la base de l'observation RMN seule de décider si le pic à $214 \mathrm{MHz}$ provient de noyaux de Co à proximité du fer ou de Co dans une phase cfc (217 MHz) éventuellement contrainte. Mais comme les mesures de diffraction $\mathrm{X}$

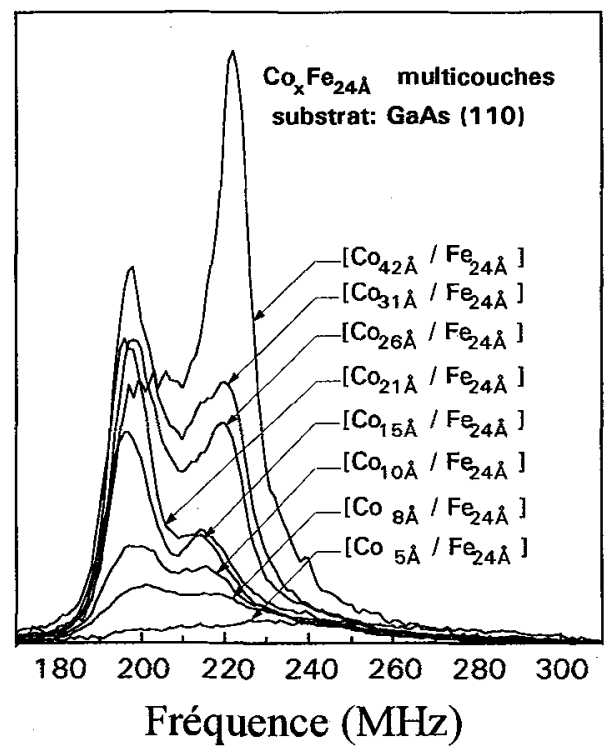

Fig.15. Spectre du Co dans les super-réseaux $\mathrm{Co} / \mathrm{Fe}$ déposés sur GaAs (110). L'aire des spectres est proportionnelle à l'épaisseur de Co déposé. 
excluent la présence d'une phase cfc ou même hc dans cette gamme d'épaisseur, la raie à $214 \mathrm{MHz}$ doit également être attribuée à du cobalt cc aux interfaces $\mathrm{Co} / \mathrm{Fe}$. Le fond étendu, quant à lui, est typique d'un alliage concentré. Au dessus de $21 \AA$ un nouveau pic crô̂t rapidement à $222 \mathrm{MHz}$ et, pour la même épaisseur, les profils de réflexion ("rocking curves") de rayons $\mathrm{X}$ montrent une dégradation abrupte de la cristallinité ainsi qu'une discontinuité dans le paramètre de réseau moyen. Ceci est associé à l'apparition d'une phase hc distordue, ou mal orientée, ou polycristalline, pour une épaisseur qui, comme indiqué en introduction, est le double de celle où cette transition est observée sur la surface libre.

Pour interpréter les spectres RMN considérons une interface parfaite entre Co et Fe dans un plan (110) de la structure cc. A l'interface les atomes de Co ont $2 \mathrm{Fe}$ proches voisins et aussi $2 \mathrm{Fe}$ en seconds voisins. L'étude des références massives montre que la fréquence de résonance du Co dans cette configuration chimique serait $216 \mathrm{MHz}$. Le satellite étroit et résolu à $214 \mathrm{MHz}$ est donc l'indication de la présence de larges zones interfaciales (110) abruptes dans le super-réseau. Cependant l'intensité contenue dans le fond spectral étendu est tout aussi considérable et prouve l'existence d'une quantité appréciable de Co dans un alliage $\mathrm{Co} / \mathrm{Fe}$ avec des configurations chimiques allant jusqu'à $8 \mathrm{Fe} \mathrm{PV}$ (Co isolé dans le fer à $290 \mathrm{MHz})$. Les couches de Co les plus fines $(5 \AA ̊)$ sont mêmes alliées à coeur.

Comme dans l'un des exemples $\mathrm{Co} / \mathrm{Cu}$ ci-dessus, aucun des deux modèles de base ne peut décrire seul la forme des spectres: le modèle d'interface abrupte ne peut reproduire la queue spectrale étendue et le modèle d'interface diffuse ne peut reproduire la lacune d'environnement entre le pic à 2 Fe PV (214 $\mathrm{MHz})$ et celui à $0 \mathrm{Fe} \mathrm{PV}(198 \mathrm{MHz})$ ou, en d'autres termes, tout profil de concentration monotone qui donne le bon nombre de configurations à 0 et $2 \mathrm{Fe} P V$ produit aussi trop de configurations à $1 \mathrm{Fe} P V$ (209 MHz). En revanche, deux modèles hybrides peuvent expliquer la forme des spectres dans la phase cc. Dans ces deux modèles la couche de Co est hétérogène et contient des grains de Co cc pur entourés d'alliage CoFe. Dans le premier modèle les grains de Co pur sont des colonnes ayant une interface abrupte avec le fer à chaque extrémité alors que dans le second les grains ont une forme pyramidale avec une interface abrupte à la base seulement. Bien que permettant une excellente reproduction des spectres expérimentaux, le premier modèle a du être rejeté; en effet, l'accord avec l'expérience est obtenu si la proportion volumique des grains purs varie de 0 à $50 \%$ lorsque l'épaisseur de Co croît de 5 à $21 \AA$; ceci est difficilement acceptable physiquement puisque cela implique que le diamètre des colonnes pures soit déterminé, dès le début du dépôt de la couche, par son épaisseur finale. Par contre le second modèle correspond beaucoup mieux à l'idée d'une croissance en îlots du Co sur une couche de fer assez plane: chaque plan atomique de la couche de cobalt est construit de plages de Co pur entourées d'alliage et les plages de Co pur sont empilées de façon à former, de plan en plan, des pyramides plus ou moins régulières. les spectres sont alors simulés par ajustement de deux paramètres par plan: i) l'aire relative occupée par les plages de Co pur (de 0 à 1: plan totalement allié à plan complet de $\mathrm{Co}$ ) et ii) la composition de la partie alliée. Les profils de ces deux paramètres, topologique et chimique, fixent les probabilités de configuration et donc la forme du spectre. Les meilleurs profils trouvés par ajustement se sont révélés superposables d'un échantillon à l'autre, indépendamment de l'épaisseur de Co déposé, ce qui montre, contrairement au cas précédent, la cohérence du modèle. La figure 16 donne un exemple d'ajustement aux spectres expérimentaux. Trois composantes principales du spectre y sont représentées: le pic principal du au Co

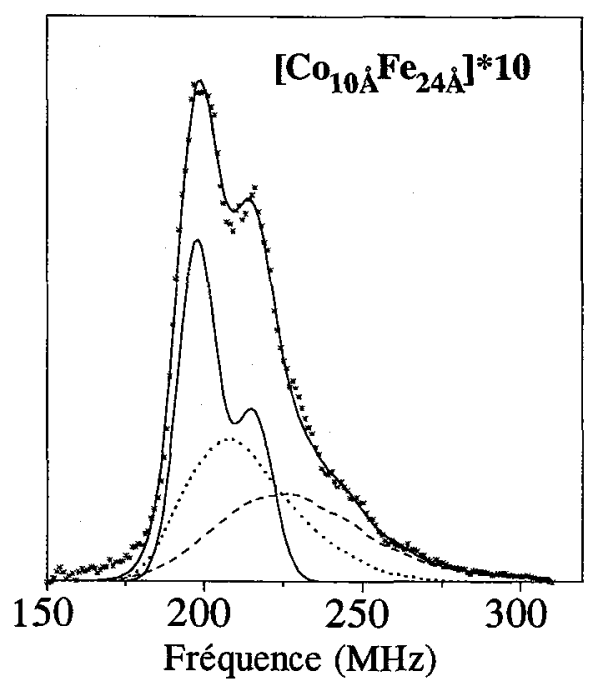

Fig.16. Spectre expérimental et simulé d'un super-réseau cc $\mathrm{Co} / \mathrm{Fe} / \mathrm{GaAs}(110)$ et contributions au spectre calculé. Trait plein: pyramides de Co cc $(198 \mathrm{MHz})$ et leur interface abrupte avec le $\mathrm{Fe}(214 \mathrm{MHz})$; pointille: interface entre Co massif et alliage CoFe; tireté: alliage $\mathrm{CoFe}$. 
cc massif et son interface abrupte avec le fer (le coeur des pyramides et leur base), l'interface entre l'alliage CoFe et le Co pur des pyramides et enfin le coeur de la partie alliée (il n'y a pas d'interface proprement dite entre le fer pur et l'alliage car son profil de composition est continu jusqu'à $100 \%$ de fer). Tous les résultats de l'étude sur cette série peuvent se résumer à l'image en coupe donnée sur la figure 17 qui représente d'une part, les observations RHEED en cours de croissance du Co et, d'autre part, les conclusions schématiques de l'étude RMN. Il faut noter que, sur cette figure, la RMN seule ne peut décider de ce qui est dessus ou dessous; un modèle de fer rugueux croissant sur du Co plat est également compatible avec l'expérience. Le choix fait sur la figure et dans la description précédente est dicté par l'observation RHEED d'une surface libre de $\mathrm{Co} / \mathrm{Fe}$ plus rugueuse que celle de $\mathrm{Fe} / \mathrm{Co}$ particulièrement au dessus de $10 \AA$ lorsqu'apparaît la phase hexagonale. En partant de la couche de fer supérieure, les profils se décrivent quantitativement de la manière suivante: 4 plans atomiques d'alliage seul dont la concentration en fer décroît rapidement de $100 \%$ à $25 \%$ (ces plans contiennent pratiquement la totalité du Co déposé pour l'échantillon à $5 \AA$ de Co). Dans les plans suivants la concentration en fer est pratiquement constante dans la partie alliée (de $25 \%$ à $20 \%$ ) tandis que la proportion de Co cc pur augmente pour atteindre $100 \%$ lorsque les bases des pyramides coalescent à $15 \AA$. Pour la plus forte épaisseur totalement cubique centrée $(21 \AA ̊)$, on atteint une épaisseur de 3 plans complets en Co cc. Au dessus de cette épaisseur critique le Co hc se développe à l'intérieur des couches de Co. Sur les $21 \AA$ maximum de Co dans une phase cubique centrée, $10-11 \AA$ sont inclus dans 1'alliage CoFe et les 11-10 $\AA$ restants sont dans des grains de Co pur dont seulement $4 \AA$ dans des plans continus plus $2 \AA$ pour l'interface $\mathrm{Co} / \mathrm{Fe}$ abrupte.

Une des conclusions de cette étude est la mise en évidence d'une épaisseur limite de $10-11 \AA$ pour le cobalt cc pur que ce soit en surface libre sur une couche de Fe ou en couche recouverte de fer: la reconstruction de la structure cc sur $10 \AA$ supplémentaires lorsque la surface est recouverte est due à l'interdiffusion entre fer et cobalt, diffusion très probablement facilitée par le caractère rugueux et mal cristallisé du Co hc de surface. Une autre conclusion intéressante est le fait que, à part dans une zone de transition où le Co est totalement allié, le cobalt cubique centré pur coexiste avec un alliage CoFe plutôt homogène et de concentration finie (20-25\%). Il se trouve que cette concentration limite est proche de celle connue pour la phase cc dans le diagramme de phase massif mais, à la différence de ce dernier dans lequel a lieu une ségrégation entre alliage cc à $25 \%$ et Co cfc, les grains de Co pur restent cc dans les multicouches (tout au moins en dessous de l'épaisseur critique). Une conclusion similaire a été tirée de l'étude d'alliages CoFe co-déposés dans des conditions semblables aux super-réseaux présentés ici [19]. 


\section{CONCLUSION}

Du fait qu'elle sonde, dans l'espace direct et à courte distance, les configurations atomiques présentes dans les matériaux, la RMN (avec les autres techniques hyperfines) complète de manière efficace les méthodes standards d'investigations structurales par diffraction, ceci particulièrement dans les systèmes inhomogènes et/ou désordonnés où la symétrie de translation est absente ou partielle. Dans cette revue non exhaustive nous avons tenté de montrer l'utilité de la technique pour l'étude de la structure des multicouches et de leurs interfaces enfouies. Compte tenu de l'information qui est également fournie sur le magnétisme local, on a vu qu'elle apporte un point de vue unique sur les corrélations entre microstructure et propriétés magnétiques telles que profils d'aimantation aux interfaces ou rigidité magnétique dans les différentes parties des structures artificielles.

\section{Remerciements}

Nous remercions vivement V. Speriosu (IBM), P. Schroeder (MSU), T. Valet (Thomson), F.J.A. den Broeder (Philips), J.P. Renard (Orsay) et leurs collaborateurs pour nous avoir fourni les échantillons de multicouches et vannes de spin $\mathrm{Co} / \mathrm{Cu}$. Le système $\mathrm{Co} / \mathrm{Fe}$ a été étudié en collaboration avec $\mathrm{G}$. Langouche et J. Dekoster (KU Leuven).

\section{Références}

[1] Akai H., Akai M., Blügel S., Drittler, B., Ebert H., Terakura K., Zeller R. et Dederichs P., Progress of Theor. Phys. Suppl. 101 (1990) 11

[2] Mény C., Jedryka E. et Panissod P., J. Phys: Cond. Matter, 5 (1993) 1547

[3] Jay J. Ph., Wojcik M. et Panissod P., Zeitschrift für Phys. B (1996, sous presse)

[4] Henry Y., Mény C., Dinia A. et Panissod P., Phys. Rev. B47 (1993) 15037

[5] de Gronckel H.A.M., Kopinga K., de Jonge W.J.M, Panissod P., Schillé J.P. et den Broeder F.J.A., Phys. Rev. B44 (1991) 9100

[6] van Alphen E.A.M., van der Heijden P.A.A et de Jonge W.J.M., J. Appl. Phys. 76 (1994) 6607

[7] van Alphen E.A.M. et de Jonge W.J.M., Phys. Rev. B51 (1995) 8182

[8] Mény C., Panissod P. et Loloee R., Phys. Rev. B45 (1992) 12269

[9] Saito Y., Inomata K., Goto A. et Yasuoka H., J. Phys. Soc. Jap. 62 (1993) 1450

[10] Valet T., Galtier P., Jacquet J.C., Mény C. et Panissod P., J. Magn. Magn. Mat. 121 (1993) 402

[11] Mény C., Panissod P., Humbert P., Nozières J.P., Speriosu V.S., Gurney B.A. et Zehringer R., J. Magn. Magn. Mat. 121 (1993) 406

[12] Mény C., Jay J.Ph., Panissod P., Humbert P., Speriosu V.S., Lefakis H., Nozières J.P. et Gurney B.A., Mat. Res. Soc. Symp. Proc. 313 (1993) 289

[13] Le Fèvre J., Chandesris D., Magnan H. et Heckmann O., J. Phys. France C9-4 (1994) 159 voir aussi N. Persat, A. Dinia, J.P. Jay, C. Mény and P. Panissod, J. Magn. Magn. Mat., (1996, sous presse)

[14] Kawakami M., J. Phys. Soc. Jap. 40 (1976) 56

[15] Houdy Ph., Boher P., Giron F., Pierre F., Chappert C., Beauvillain P., Le Dang K., Veillet P. et Velu E., J. Appl. Phys. 69 (1991) 5667

[16] Dekoster J., Jedryka E., Mény C. et Langouche G., J. Magn. Magn. Mater. 121 (1993) 69 et Europhys. Lett. 22 (1993) 433

[17] Dekoster J., Jedryka E., Wójcik M. et G. Langouche, J. Magn. Magn. Mater. 126 (1993) 12

[18] Jay J. Ph., Jedryka E., Wojcik M., Dekoster J., Langouche G. et Panissod P., Zeitschrift für Phys. B (1996, sous presse)

[19] Wojcik M., Jay J. Ph., Panissod P., Jedryka E., Dekoster J., Langouche G., Digest de ICMFS'94 et à publier. 